\title{
Comparison of Linear and Conic Power Flow Formulations for Unbalanced Low Voltage Network Optimization
}

\author{
Marta Vanin ${ }^{* \dagger}$, Hakan Ergun*†, Reinhilde D'hulst ${ }^{\S \dagger}$ and Dirk Van Hertem* ${ }^{* \dagger}$ \\ ${ }^{*}$ KU Leuven ESAT - Electa, Kasteelpark Arenberg 10, 3001 Heverlee, Belgium \\ $\dagger$ EnergyVille, Thor Park, Poort Genk 8310, 3600, Genk, Belgium \\ $\S$ VITO, Boeretang 200, 3400 Mol, Belgium
}

\begin{abstract}
The electrification of transport and heating systems brings new challenges for the low voltage distribution networks, which in the future might be more prone to congestion. This calls for methods to perform optimization and control actions, to ensure their correct operation. Their large extension and non-negligible degree of voltage unbalance make low voltage systems difficult to analyze. This paper features a comparison of three unbalanced formulations for power flow and OPF analyses: the full nonlinear, non-convex AC formulation, a second-order cone relaxation and a linear approximation. Accuracies and computational efforts are evaluated for different problem types, to assess whether the conic and linear formulation are suitable to perform low voltage systems calculations.
\end{abstract}

Index Terms-Distribution networks; mixed-integer programming; optimal power flow; voltage unbalance

\section{NOMENCLATURE}

Sets

$\mathcal{N} \quad$ Set of feeder buses

$E \quad$ Set of branches in the feeder, forward orientation

$E^{R} \quad$ Set of branches in the feeder, reverse orientation

$L, L_{i} \quad$ Set of loads and loads at bus $i$

$S, S_{i} \quad$ Set of shunts and shunts at bus $i$

$G, G_{i} \quad$ Set of generators and generators at bus $i$

$P V, P V_{i} \quad$ Set of $\mathrm{PV}$ generators and $\mathrm{PV}$ gen. at bus $i$

$\phi \quad$ Set of conductors

The sets $G, G_{i}$ include both PV generators and the feeder transformer substation $(S S)$, such that $G=P V \cup S S ; G_{i}=$ $P V_{i} \cup S S$.

In the present analysis, the feeder data used are Kronreduced, thus $\phi$ corresponds to the feeder phases. Let $|\phi|$ be the cardinality of $\phi$, the following variables and parameters are used:

Variables and parameters

$\begin{array}{ll}\mathbf{U}_{i}:=\left[U_{i, c}\right]_{c \in \phi} & \text { Voltage phasor at bus } i \\ \mathbf{U}^{s s}:=\left[U_{c}^{s s}\right]_{c \in \phi} & \text { Voltage phasor at transformer substa- } \\ & \text { tion } \\ \mathbf{U}^{r}:=\left[U_{c}^{r}\right]_{c \in \phi} & \text { Reference voltage phasor } \\ \mathbf{I}_{i j}^{s}:=\left[I_{i j, c}^{s}\right]_{c \in \phi} & \text { Series current at branch }(i, j)\end{array}$

This work is supported by the Flemish DSO Fluvius in the framework of the research project ADriaN - Active Distribution Networks.
$\mathbf{S}^{s s}:=\left[S_{c}^{s s}\right]_{c \in \phi} \quad$ Apparent power injection at the substation

$\mathbf{S}_{k}^{g}:=\left[S_{k, c}^{g}\right]_{c \in \phi} \quad$ Apparent power generation at generator $k$

$\mathbf{S}_{k}^{d}:=\left[S_{k, c}^{d}\right]_{c \in \phi}$ Apparent power demand at load $k$

$\mathbf{S}_{i j} \in \mathbb{C}^{|\phi| \times|\phi|}$

Total apparent power flow at branch $(i, j)$

$\mathbf{S}_{i j}^{s} \in \mathbb{C}^{|\phi| \times|\phi|} \quad$ Series apparent power flow at branch $(i, j)$

$\mathbf{Y}_{k}^{s} \in \mathbb{C}^{|\phi| \times|\phi|} \quad$ Shunt admittance of shunt $k$

$\mathbf{Y}_{i j}^{c}, \mathbf{Y}_{j i}^{c} \in \mathbb{C}^{|\phi| \times|\phi|} \operatorname{Branch}(i, j)$ pi-section shunt admittance

$\mathbf{Y}_{i j} \in \mathbb{C}^{|\phi| \times|\phi|} \quad$ Branch $(i, j)$ pi-section series admit-

$\mathbf{Z}_{i j}=\mathbf{Y}_{i j}^{-1}$

$\underline{u}_{i, c}, \bar{u}_{i, c} \in \mathbb{R}$ tance

Series impedance of branch $(i, j)$

Lower and upper bound of voltage magnitude of bus $i$, phase $c$

$\underline{s}_{k, c}^{g}, \bar{s}_{k, c}^{g} \in \mathbb{C}$

$\bar{s}_{i j, c} \in \mathbb{R}$

$c_{0 k}, c_{1 k} \in \mathbb{R}$

Lower and upper bound of apparent generation at generator $k$, phase $c$ Thermal limit of branch $(i, j)$, phase $c$ Cost coefficients of generator $k$

Vectors and matrices are displayed in bold. The notation $\mathbf{x}_{i}:=\left[x_{i, c}\right]_{c \in \phi}$ shows that $\mathbf{x}_{i}$ is a vector built by putting together the phase values $x_{i, c}$. Finally, the following operators are used:

Operators

$(\cdot)^{H} \quad$ Takes the Hermitian transpose of a matrix

$\operatorname{diag}(\cdot) \quad$ Takes the diagonal of a matrix as a vector

$\mathfrak{R}(\cdot) \quad$ Takes the real component of a complex number

\section{INTRODUCTION}

Low voltage distribution networks (LVDNs) in Europe have received limited attention in the past. This is because traditional residential user loads are relatively small and the infrastructure was developed to be robust enough to operate safely and provide adequate quality of service.

However, LVDNs are now facing the integration of distributed generation (rooftop PV systems), electric transport and electric heating, loads having a power demand often comparable to or even higher than that of a typical European 
household [1]. This results in higher degrees of voltage unbalance, if these devices are connected on a single phase, and increased risk for congestion and power quality issues, which might affect the correct operation of the networks.

Thus, both deeper understanding of the impact of these technologies on existing feeders and methods to perform optimization and control actions [2] are needed, so that distribution system operators (DSOs) can ensure quality of power supply.

The physical characteristics of the low voltage system, together with its large extension (hundreds of thousands of feeders), makes it difficult to perform power flow (PF) and optimal power flow (OPF) analyses with the classic methods used, for example, in transmission networks.

To make specific problems tractable or solvable in acceptable time, various approximations and relaxations of the power flow equations have been derived, for both the balanced and unbalanced case [3], [4], [5]. A comprehensive overview can be found in [6]. However, it is not always clear which formulation is preferable given a real-life problem. Comparisons of different formulations for different specific problems exist in the literature [7], [8], [9], but results are often only presented on one or few test feeders. Furthermore, to the best of the authors' knowledge, no comparisons are present in the literature that address multiple unbalanced formulations on a variety of problems.

This paper analyzes the performance of the linear approximation known as "simplified DistFlow" (SDF) [3], and the second-order cone (SOC) chordal conversion-based relaxation [10] of the positive semi-definite relaxation from [3] on a large number of real-life feeders. Both formulations are compared with a nonlinear non-convex (AC) formulation [11]. The simplified formulations are chosen as they present a limited number of simplifying assumptions compared to other available in the literature, thus promising high accuracy.

\section{A. Contributions}

Performing empirical and analytical error analyses of the different existing power flow formulations is an open research topic [6], bottlenecked by the lack of publicly available power systems test cases. Furthermore, not all existing test cases represent real-life conditions in sufficient detail [12], [13].

In this work, the formulations have been tested on a large number of real-life low voltage radial distribution feeders and power profiles, made available by the Flemish DSO Fluvius. To the best of the authors' knowledge, this is the first time that these formulations are benchmarked on a large number of real-life feeders. Also, the analysis takes into account how the number of nodes and the degree of unbalance affect the accuracy and computational effort of the formulations. In particular, phase unbalance is expected to compromise the accuracy of the SDF formulation, due to the "small unbalance" assumption in its derivation. One of the aims of this work is to assess whether the accuracy is still reasonable for unbalance levels which are considered acceptable in LVDNs. To assess the accuracy and speed, the formulations are compared using a variety of PF, OPF and mixed-integer OPF.
To make sure that the formulations are adequately compared, they share a common implementation structure and software base: PowerModelsDistribution.jl [14] is used, an open-source Julia package for the exploration of different power flow formulations for distribution networks [15]. The SDF approximation is chosen because it includes resistive losses, reactive power and voltage sag. SDF and SOC are derived starting from the same branch flow relaxation model. Branch flow relaxations present numerical advantages over the bus injection model, which are especially evident in threephase models [3], [16].

It should be noted that the AC formulation from [14], used in the current work, has been validated with the wellestablished OpenDSS [17] in reference [18]. This allows the authors of the present paper to focus on benchmarking the SDF and SOC against the already validated AC formulation.

Simplified power flow formulations are used to solve problems which are particularly challenging because they are unbalanced [19], multiperiod [20], [21] or have mixed-integer variables [22], [23]. Section IV describes and tries to tackle a problem that features all these criticalities at once.

Section II presents the indices which are used to assess the performance of the different formulations. Section III provides the PF and OPF problem formulations and compares them for the indices defined in Section II. Finally, Section IV presents an OPF-based congestion management strategy with mixedinteger variables.

\section{PERFormanCE INDICES}

In this section, parameters and considerations used to compare the different formulations are introduced.

\section{A. Accuracy}

In the power flow problem, where there is only one control variable (the slack bus at the root of the feeder), the accuracy is compared based on the voltage magnitudes, whereas in OPF problems the optimality gap can be used as an additional indicator.

1) Voltage magnitude accuracy: Let $\mathcal{N}$ be the set of buses in a feeder and $N$ be the cardinality of $\mathcal{N}$. For each feeder, the average $\left(\Delta \mathrm{U}_{\mathrm{avg}}\right)$ and the maximum $\left(\Delta \mathrm{U}_{\max }\right)$ voltage magnitude difference over the buses are used to evaluate the accuracy, such that, for the SDF case:

$$
\begin{array}{r}
\Delta \mathrm{U}_{\mathrm{avg}, \mathrm{SDF}}=\frac{1}{\mathrm{~N}} \sum_{\mathrm{i} \in \mathcal{N}}\left|\mathrm{U}_{\mathrm{AC}_{\mathrm{i}}}-\mathrm{U}_{\mathrm{SDF}_{\mathrm{i}}}\right| \quad[\mathrm{p} . \mathrm{u} .] \\
\Delta \mathrm{U}_{\max , \mathrm{SDF}}=\max \left\{\left|\mathrm{U}_{\mathrm{AC}_{\mathrm{i}}}-\mathrm{U}_{\mathrm{SDF}_{\mathrm{i}}}\right|\right\}_{\mathrm{i} \in \mathcal{N}} \quad[\mathrm{p} . \mathrm{u} .],
\end{array}
$$

where $\mathrm{U}_{\mathrm{AC}, \mathrm{i}}$ is the voltage magnitude at bus $i$, obtained with the non-convex $\mathrm{AC}$ formulation. Similar expressions are derived for the SOC formulation ( $\left.\Delta \mathrm{U}_{\mathrm{avg}, \mathrm{SOC}}, \Delta \mathrm{U}_{\max , \mathrm{SOC}}\right)$.

2) Optimality gap: The difference in the objective values, or optimality gap, $(\Delta \mathrm{Obj})$ is calculated for every feeder and is expressed in percentual values, as follows:

$$
\begin{aligned}
& \Delta \mathrm{Obj}_{\mathrm{SDF}}=\left(\mathrm{Obj}_{\mathrm{AC}}-\mathrm{Obj}_{\mathrm{SDF}}\right) / \mathrm{Obj}_{\mathrm{AC}} \times 100[\%] \\
& \Delta \mathrm{Obj}_{\mathrm{SOC}}=\left(\mathrm{Obj} \mathrm{AC}_{\mathrm{AC}}-\mathrm{Obj}_{\mathrm{SOC}}\right) / \mathrm{Obj}_{\mathrm{AC}} \times 100[\%]
\end{aligned}
$$




\section{B. Voltage unbalance}

An important assumption underlying the simplified formulations is that the voltages are nearly balanced. It is thus of concern whether they are still representative for feeders which present a high number of large single-phase loads.

The voltage unbalance definition chosen for this study is the Voltage Unbalance Factor (VUF) by IEC [24], which includes in its definition both phase angle and voltage magnitude unbalance. It is also adopted as a reference by CENELEC [25] as indicator of power quality. The VUF is defined for every bus, as follows.

Let $\mathbf{U}_{a}, \mathbf{U}_{b}$ and $\mathbf{U}_{c}$ be the line-to-ground voltage phasors of the three-phases and $\mathbf{a}=e^{i \cdot 2 / 3 \pi}$ where $\mathbf{U}_{n}$ and $\mathbf{U}_{p}$ are, respectively, the negative and the positive sequence phasors:

$$
\begin{gathered}
\mathbf{U}_{p}=\frac{\mathbf{U}_{a}+\mathbf{a} \mathbf{U}_{b}+\mathbf{a}^{2} \mathbf{U}_{c}}{3} \\
\mathbf{U}_{n}=\frac{\mathbf{U}_{a}+\mathbf{a}^{2} \mathbf{U}_{b}+\mathbf{a} \mathbf{U}_{c}}{3} \\
\operatorname{VUF}[\%]=\frac{\left|\mathbf{U}_{n}\right|}{\left|\mathbf{U}_{p}\right|} \times 100
\end{gathered}
$$

According to [25], acceptable power quality is suppplied when the VUF is below $2 \%$ for $95 \%$ of the week (mean 10 minutes rms values). Several figures in this paper make use of $\mathrm{VUF}_{\text {max }}$, which is defined for every feeder as:

$$
\mathrm{VUF}_{\text {max }}=\max \left\{\mathrm{VUF}_{\mathrm{i}}\right\}_{\mathrm{i} \in \mathcal{N}}[\%]
$$

Currently, distribution feeders do not show significant amount of unbalance. To allow analyses for higher degrees of unbalance, it is assumed that $30 \%$ of the connection points feature an electric vehicle (EV), which is exclusively charging at home using a single-phase charger of $3.3 \mathrm{kVA}$ (16 A). More details about the charging behaviour model can be found in [26]. Each low voltage feeder responds differently to the addition of these devices, depending on its topology and connected customers.

It is worth noting that there are considerable differences between European and American (US) grids, which affect, for instance, the way phase unbalance is caused. In European feeders, like the ones used in this paper, the principal cause of unbalance are unevenly distributed single-phase loads, while in the US this is caused by the grid layout itself [27]. It is not to be excluded that the impact of unbalance on US feeders on the accuracy of (optimal) PF calculations can be different from the European case presented here.

\section{Solvers and computational time}

Table I illustrates the total computational time to solve PF and OPF problems on the 500 used feeders, using different solvers. All calculations described in this work are performed on a 64-bit machine with Intel(R) Xeon(R)CPU E5-4610 v4 @ $1.80 \mathrm{GHz}$ and $32 \mathrm{~GB}$ RAM. It should be noted that of the tested solvers, only Ipopt [28] can solve non-convex problems, while the others are not capable (n.c.). For Ipopt, two different underlying linear solvers have been tested: the default, MUMPS, and the HSL MA 27 [29].
TABLE I: Total computational time to solve $500 \mathrm{OPF}$ problems with different solvers and formulations

\begin{tabular}{l||c|c|c}
\hline Solver & SDF [s] & SOC [s] & AC [s] \\
\hline Cplex v12.7 & 6.46 & 111.59 & n.c. \\
Mosek v8.1.0.80 & 4.67 & 68.68 & n.c. \\
Gurobi v9.0.1 & 2.56 & 86.81 & n.c. \\
Ipopt v3.12.10 - MUMPS & 25.36 & 3241.46 & 34.06 \\
Ipopt v3.12.10 - MA27 & 10.41 & 2151.75 & 20.84 \\
\hline
\end{tabular}

The computational results are displayed for the best performing solver: Gurobi for SDF calculations, Mosek for SOC, and Ipopt with linear solver MA27 for AC. The initial values provided for the variables are the same for every solver: unitary voltage magnitude and balanced phase angles at every bus and, when it is a variable, PV generation equal to the maximum instantaneous PV injection.

\section{Problems With CONTINUOUS VARiables}

This section deals with the formulation and results of problems that only feature continuous variables. The results refer to simulations on 500 Belgian low voltage feeders.

\section{A. Power flow analysis}

Given fixed demand and generation along the feeder, the PF problem consists of finding voltage values for all buses (and branch currents). The problem can be expressed mathematically with a bus injection model (BIM) or a branch flow model (BFM) [14]. The mathematical expressions for the AC version of both BIM and BFM are reported below. These provide a complete conceptual explanation that also applies to the other formulations. Due to space limitations, the reader is referred to [3], [10], [11] and [14] for the details on the implementation and the derivation of the SOC and SDF formulations. The AC implementation used in this work is a BIM, while the SDF and SOC are BFMs by construction.

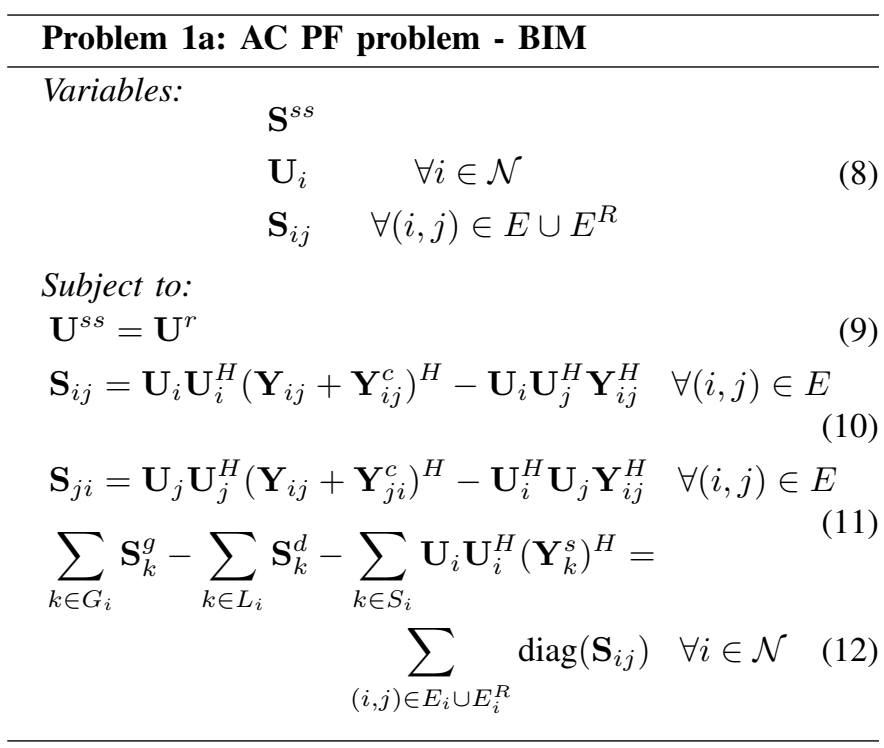




\section{Problem 1b: AC PF problem - BFM}

Variables:

$$
\begin{aligned}
& \text { Variables (8) and: } \\
& \mathbf{I}_{i j}^{s} \quad \forall(i, j) \in E
\end{aligned}
$$

\section{Subject to:}

Constraints (9) and (12), and:

$$
\begin{array}{ll}
\mathbf{U}_{j}=\mathbf{U}_{i}-\mathbf{Z}_{i j} \mathbf{I}_{i j}^{s} & \forall(i, j) \in E \cup E^{R} \\
\mathbf{S}_{i j}^{s}=\mathbf{U}_{i}\left(\mathbf{I}_{i j}^{s}\right)^{H} & \forall(i, j) \in E \cup E^{R} \\
\mathbf{S}_{i j}=\mathbf{S}_{i j}^{s}+\mathbf{U}_{i} \mathbf{U}_{i}^{H}\left(\mathbf{Y}_{i j}^{c}\right) & \forall(i, j) \in E \cup E^{R} \\
\mathbf{S}_{i j}+\mathbf{S}_{j i}=\mathbf{U}_{i} \mathbf{U}_{i}^{H}\left(\mathbf{Y}_{i j}^{c}\right)^{H}+ & \\
\mathbf{Z}_{i j} \mathbf{I}_{i j}^{s}\left(\mathbf{I}_{i j}^{s}\right)^{H}+\mathbf{U}_{j} \mathbf{U}_{j}^{H}\left(\mathbf{Y}_{j i}^{c}\right)^{H} & \forall(i, j) \in E
\end{array}
$$

Equation (9) indicates that the voltage phasor at the substation is used as fixed reference. Specifically, in the present analysis, the voltage magnitude at the substation is the nominal and the phase angles are equally spaced from each other. Equations (10)-(11) apply Ohm's law along the branches. Equation (12) enforces power balance in each bus. In the BFM, (10)-(11) are replaced by (14)-(17). Equation (14) represents the voltage drop (and losses) along the branch, (15) associates the series power flow to the series current variable, and (16) shows the relation between total and series power flow, respectively. Finally, (17) links the losses to the power flows.

Demand values $\mathbf{S}_{k}^{d} \quad \forall k \in L_{i}$ in (12) are fixed values, together with the PV generation $\mathbf{S}_{k}^{g} \forall k \in P V_{i}$. The power injection at the substation, $\mathbf{S}^{s s}=\mathbf{S}_{k}^{g} \forall k \in G_{i} \backslash P V_{i}$, on the other hand, is a variable.

Fig. 1 shows that $\Delta U_{a v g, S D F}$ and $\Delta U_{\max , S D F}$ increase with increasing $\mathrm{VUF}_{\max }$ : the voltage magnitudes deviate more from the AC solution, as expected. Due to the construction of the SOC itself, the absence of an objective function makes any of the values allowed by the relaxation feasible, without any guarantee on the voltage accuracy. Therefore, the use of SOC relaxations does not make sense for the classic PF problem.

Section III-B shows that it is possible to add a simple objective function that makes the SOC usable without affecting the results for the other formulations. Therefore, considerations on the performance of both SOC and SDF for PF problems are carried out in Section III-B.

\section{B. Power flow analysis with objective function}

Equation (20) adds an objective function representing the minimization of the generation cost to the PF problem, which means that the minimum amount of power injected by the slack node is sought. It should be noted that the cost is here modeled as a linear function of power generation. This assumption allows to use the same objective with all the different formulations, keeping the SDF implementation linear. The actual objective has little importance for the purpose of this paper.

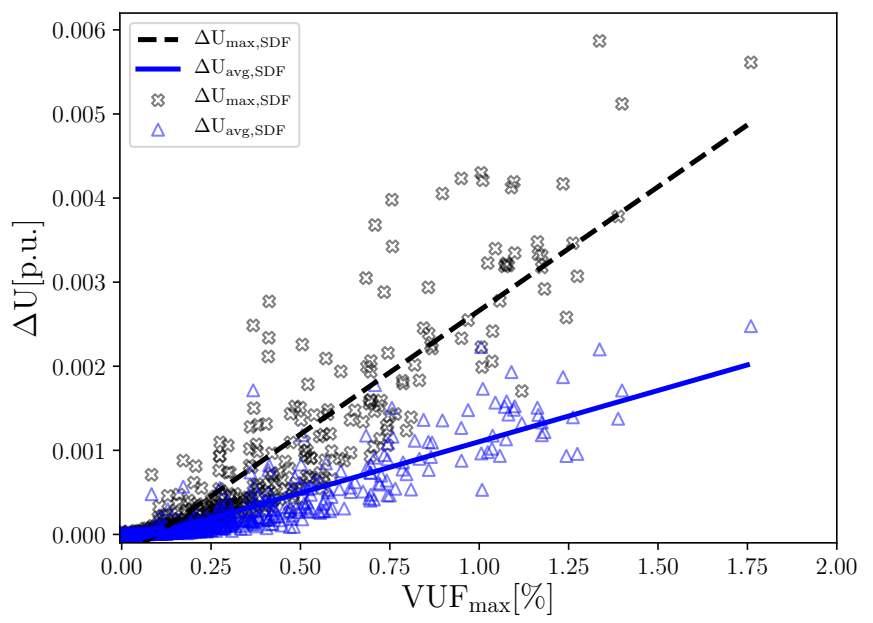

Fig. 1: Voltage magnitude accuracy decreases with increasing unbalance, $\mathrm{PF}$ problem, SDF formulation

\section{Problem 2: AC PF with objective - BIM and BFM Variables: \\ (8) in the BIM; (8) and (13) in the BFM \\ $\mathbf{S}_{k}^{g} \quad \forall k \in P V$}

Subject to:

$$
\begin{aligned}
& \text { (9) - (12) in the BIM } \\
& \text { (9),(12) and (14) - (17) in the BFM } \\
& S_{k, c}^{g}=\bar{s}_{k, c}^{g} \quad \forall k \in P V, c \in \phi
\end{aligned}
$$

With objective:

$$
\operatorname{minimize} \sum_{k \in G} c_{1 k}\left(\sum_{c \in \phi} \mathfrak{R}\left(S_{k, c}^{g}\right)\right)+c_{0 k}
$$

Generation needs to be declared as variable (18), as it is present in the objective. Adding (18) and (20) to the AC or SDF problem does not affect the results, as long as (18) is forced to be equal to the instantaneous PV injection $\bar{s}_{k, c}^{g}$, in (19). Adding the objective function prevents the SOC relaxation from creating unrealistic power losses along the feeder, making the formulation acceptable for this PF problem.

The accuracy results are reported in Fig. 2. It is observed that both the SDF and SOC are less accurate for higher degrees of voltage unbalance. However, the quality of the SDF formulation is very high even in feeders in which the voltage unbalance level is close to the maximum allowed by the standard [25]. $\Delta \mathrm{U}_{\mathrm{avg}, \mathrm{SDF}}$ and $\Delta \mathrm{U}_{\mathrm{max}, \mathrm{SDF}}$ never exceed 0.0025 p.u. and 0.0059 p.u., respectively, which correspond to phase voltage differences of barely $0.58 \mathrm{~V}$ and $1.4 \mathrm{~V}$ in a $230 \mathrm{~V}$ distribution feeder.

Interestingly, the SDF approximation is in general more accurate than the SOC relaxation. $\Delta \mathrm{U}_{\max , \mathrm{SDF}}$ is always lower than $\Delta \mathrm{U}_{\mathrm{max}, \mathrm{SOC}}$, and on average 0.0001 p.u. better. On the other hand, $\Delta \mathrm{U}_{\mathrm{avg}, \mathrm{SDF}}$ is on average only $4 \cdot 10^{-5}$ p.u. better than $\Delta \mathrm{U}_{\mathrm{avg}, \mathrm{SOC}}$, and for $\mathrm{VUF}_{\max } \geq 1 \%, \Delta \mathrm{U}_{\mathrm{avg}, \mathrm{SDF}}$ is higher than $\Delta \mathrm{U}_{\mathrm{avg}, \mathrm{SOC}}$ in $73 \%$ of the cases. 


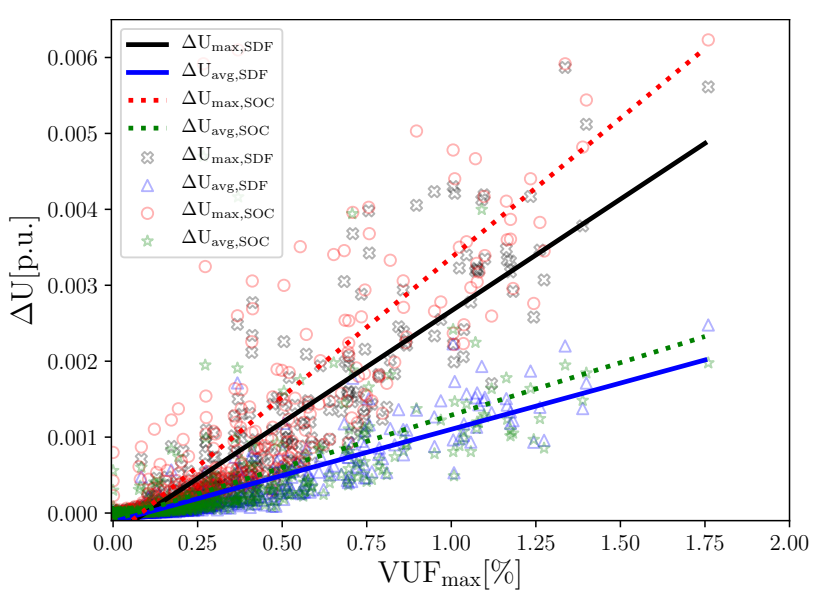

Fig. 2: Voltage magnitude accuracy decrease with increasing unbalance, $\mathrm{PF}$ problem with objective function

TABLE II: Parameters for linear trend lines of $\Delta \mathrm{U}_{\mathrm{avg}, \mathrm{SDF}}$, $\Delta \mathrm{U}_{\text {max }, \mathrm{SDF}}, \Delta \mathrm{U}_{\mathrm{avg}, \mathrm{SOC}}, \Delta \mathrm{U}_{\mathrm{max}, \mathrm{SOC}}$ of Fig. 2

\begin{tabular}{ccccc}
\hline $\mathrm{f}(\mathrm{y})$ & $\mathrm{x}$ & $p_{1}$ & $p_{0}$ & $E$ \\
\hline$\Delta \mathrm{U}_{\text {avg,SDF }}$ & $\mathrm{VUF}_{\max }$ & 0.0012 & -0.0001 & $1.77 \mathrm{E}-5$ \\
$\Delta \mathrm{U}_{\max , \mathrm{SDF}}$ & $\mathrm{VUF}_{\max }$ & 0.0029 & -0.0003 & $9.13 \mathrm{E}-5$ \\
$\Delta \mathrm{U}_{\text {avg,SOC }}$ & $\mathrm{VUF}_{\max }$ & 0.0014 & $9.01 \mathrm{E}-5$ & $1.60 \mathrm{E}-4$ \\
$\Delta \mathrm{U}_{\text {max }, S O C}$ & $\mathrm{VUF}_{\max }$ & 0.0037 & -0.0003 & $5.44 \mathrm{E}-4$ \\
\hline
\end{tabular}

To help visualize these small differences and the relative performance of the formulations, linear trend lines of the scattered results are shown in Fig. 2. The lines have structure $f(x)=p_{1} \cdot x+p_{0}$ and are obtained by minimizing the squared error $E:=\sum_{j \in \mathcal{D}}\left|f\left(x_{j}\right)-y_{j}\right|^{2}$, where $\mathcal{D}$ is the set of the data points, and $x_{j}, y_{j}$ are the data point coordinates. The values of the coefficients can be found in Table $\mathrm{V}$.

It was investigated whether there is a clearer correlation between voltage accuracy and number of buses in the feeder. However, the high number of feeders with less than 100 buses and low accuracy in Fig. 3 shows that there is a lower correlation than in the case of $\mathrm{VUF}_{\max }$. This is also shown by the higher $E$ values of the trend lines for Fig. 3 in Table VI with respect to those in Table V.

Finally, Fig. 4 shows the computational effort with the chosen solvers. Solving the SDF formulation is on average 4.86 times faster than the $\mathrm{AC}$ formulation and 22.5 times faster than the SOC formulation. In both cases, the difference increases for feeders with more buses. In particular, the slope of the AC trend line in Fig. 4 is 23.9 times steeper than that of the SDF. It is somehow surprising that SOC solutions are found slower than $\mathrm{AC}$ solutions, given the lower computational complexity of SOC programming. This might be caused by the different maturity of the used solvers or numerical issues, and has occurred also for other test cases [18]. One possible explanation could be the unsuitability of the application of the presented SOC to the feeder topologies used in this analysis. The SOC solver time is better represented by a trend of the form $f(x)=p_{2} \cdot x^{2}+p_{1} \cdot x+p_{0}$.

To conclude, despite presenting good accuracy, the SOC relaxation performs worse than the SDF on average, especially

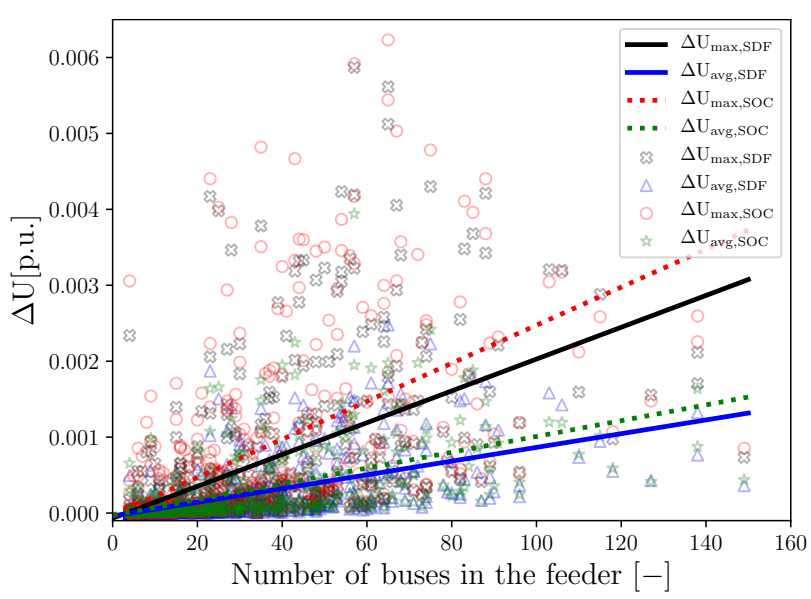

Fig. 3: Voltage magnitude accuracy in function of number of buses, PF problem with objective function

TABLE III: Parameters for linear trend lines of $\Delta \mathrm{U}_{\mathrm{avg}, \mathrm{SDF}}$, $\Delta \mathrm{U}_{\text {max }, \mathrm{SDF}}, \Delta \mathrm{U}_{\mathrm{avg}, \mathrm{SOC}}, \Delta \mathrm{U}_{\mathrm{max}, \mathrm{SOC}}$ of Fig. 3

\begin{tabular}{ccccc}
\hline $\mathrm{f}(\mathrm{y})$ & $\mathrm{x}$ & $p_{1}$ & $p_{0}$ & $E$ \\
\hline$\Delta \mathrm{U}_{\text {avg,SDF }}$ & Number of buses & $9.04 \mathrm{E}-6$ & $-3.90 \mathrm{E}-5$ & $5.94 \mathrm{E}-5$ \\
$\Delta \mathrm{U}_{\max , \mathrm{SDF}}$ & Number of buses & $2.10 \mathrm{E}-5$ & $-6.73 \mathrm{E}-5$ & $3.51 \mathrm{E}-4$ \\
$\Delta \mathrm{U}_{\text {avg,SOC }}$ & Number of buses & $1.04 \mathrm{E}-5$ & $-3.08 \mathrm{E}-5$ & $1.66 \mathrm{E}-4$ \\
$\Delta \mathrm{U}_{\max , \mathrm{SOC}}$ & Number of buses & $2.51 \mathrm{E}-5$ & $-3.20 \mathrm{E}-5$ & $8.56 \mathrm{E}-4$ \\
\hline
\end{tabular}

when $\Delta \mathrm{U}_{\max }$ is considered. This, together with the lack of computational advantage over the other formulations, makes the use of the SOC questionable for this type of PF problems. In the SDF case, on the other hand, the compromise between speed and accuracy can be worth considering, depending on the intended use (e.g. real-time or long-term planning) and on the existing knowledge of the physical parameters of the distribution feeders, which are often only roughly known. In any case, the accuracy of the SDF has proven remarkable for all the analyzed feeders.

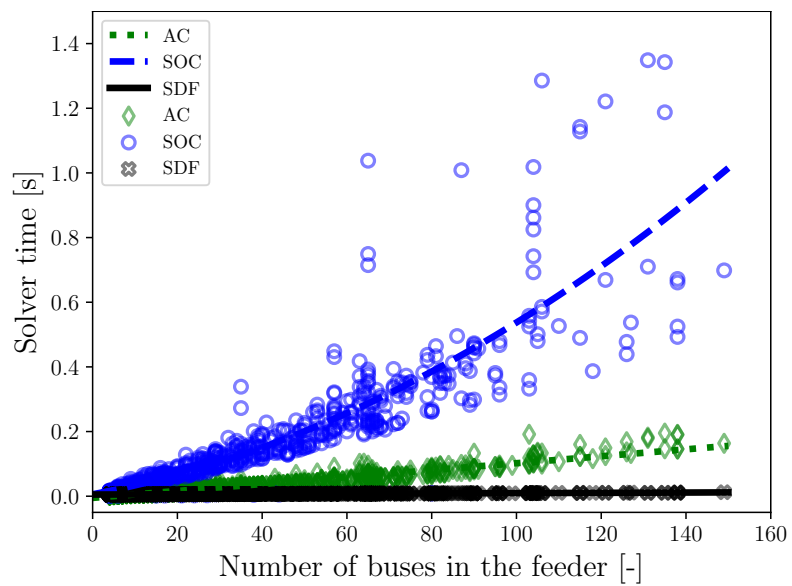

Fig. 4: Solver times for the different formulations, PF problem with objective function 
TABLE IV: Parameters for trend lines of AC, SOC and SDF solver times in Fig. 4

\begin{tabular}{cccccc}
\hline $\mathrm{f}(\mathrm{y})$ & $\mathrm{x}$ & $p_{2}$ & $p_{1}$ & $p_{0}$ & $E$ \\
\hline AC & Number of buses & - & $1.07 \mathrm{E}-3$ & $-5.22 \mathrm{E}-3$ & 0.062 \\
SOC & Number of buses & $2.83 \mathrm{E}-5$ & $2.48 \mathrm{E}-3$ & $5.91 \mathrm{E}-3$ & 7.604 \\
SDF & Number of buses & - & $4.48 \mathrm{E}-5$ & $5.06 \mathrm{E}-3$ & $5.05 \mathrm{E}-5$ \\
\hline
\end{tabular}

\section{OPF analysis}

To conclude the section on continuous variable problems, an OPF problem is presented. In addition to the objective function (20), all PV generators are made dispatchable and branch flow and voltage magnitude limits are applied.

\section{Problem 3: AC OPF - BIM and BFM}

Variables:

(8) and (18) in the BIM

(8), (13) and (18) in the BFM

Subject to:

(9) - (12) in the BIM

(9), (12) and (14) - (17) in the BFM

$\underline{u}_{i, c} \leq\left|U_{i, c}\right| \leq \bar{u}_{i, c}$

$\underline{s}_{k, c}^{g} \leq S_{k, c}^{g} \leq \bar{s}_{k, c}^{g}$

$\left|\operatorname{diag}\left(\mathbf{S}_{i j}\right)\right| \leq \bar{s}_{i j}$

With objective:

(20)

The voltage limits in (21) are defined by the grid standards, while the thermal limits (23) depend on the cable properties. In the present implementation, the upper limit for the generators (22) is the instantaneous generated power, while the lower limit is zero.

Fig. 5 shows the accuracy of SDF and SOC for the presented OPF problem. The observations are in line with the PF analysis: increase in unbalance decreases the accuracy of simplified formulations, and SDF performs slightly better, especially when it comes to $\Delta \mathrm{U}_{\max }$ values. There is no major difference between the accuracy trend lines of Fig. 2 and Fig. 5 , although both formulations' accuracies are slightly higher in the OPF problem.

The computational efforts, on the other hand, present more significant differences. Fig. 6 shows that although the AC formulation remains faster than the SOC, the difference has reduced w.r.t. the PF problem. It can be observed that although the slope of the trend line in the AC case has not substantially changed, a higher number of outlier values are present. Furthermore, the solver time for the SOC has substantially decreased. This is likely attributed to the presence of bounds on the variables (e.g. (21)), which contribute to reducing the search space. Further tightening the bounds could lead to better computational performance, but results in the risk of losing feasible solutions if the search space is reduced too much.

Given its better accuracy and shorter solution time, it seems preferable to resort to the SDF formulation for the presented type of problems, instead of tightening the bounds of the SOC.

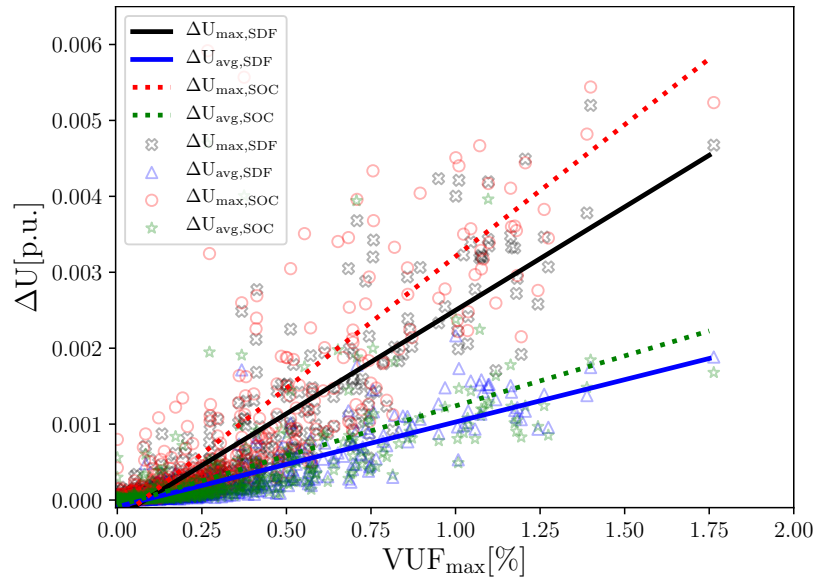

Fig. 5: Voltage magnitude accuracy decrease with increasing unbalance, OPF problem

TABLE V: Parameters for linear trend lines of $\Delta \mathrm{U}_{\mathrm{avg}, \mathrm{SDF}}$, $\Delta \mathrm{U}_{\text {max }, \mathrm{SDF}}, \Delta \mathrm{U}_{\mathrm{avg}, \mathrm{SOC}}, \Delta \mathrm{U}_{\mathrm{max}, \mathrm{SOC}}$ of Fig. 5

\begin{tabular}{ccccc}
\hline $\mathrm{f}(\mathrm{y})$ & $\mathrm{x}$ & $p_{1}$ & $p_{0}$ & $E$ \\
\hline$\Delta \mathrm{U}_{\text {avg,SDF }}$ & $\mathrm{VUF}_{\max }$ & 0.0012 & $-8.77 \mathrm{E}-5$ & $1.65 \mathrm{E}-5$ \\
$\Delta \mathrm{U}_{\max , \mathrm{SDF}}$ & $\mathrm{VUF}_{\max }$ & 0.0027 & $-2.25 \mathrm{E}-4$ & $8.59 \mathrm{E}-5$ \\
$\Delta \mathrm{U}_{\text {avg,SOC }}$ & $\mathrm{VUF}_{\max }$ & 0.0013 & $-7.63 \mathrm{E}-5$ & $1.57 \mathrm{E}-4$ \\
$\Delta \mathrm{U}_{\max , \mathrm{SOC}}$ & $\mathrm{VUF}_{\max }$ & 0.0035 & $-2.61 \mathrm{E}-4$ & $5.21 \mathrm{E}-4$ \\
\hline
\end{tabular}

The SDF is on average 4.02 times faster than the $\mathrm{AC}$ formulation and 13.65 times faster than the SOC formulation, respectively. The slopes of the SOC and AC trend lines in Fig. 6 are, respectively, 20.54 and 5.46 times steeper than that of the SDF, showing again that the use of the SDF is the more advantageous in feeders with more buses.

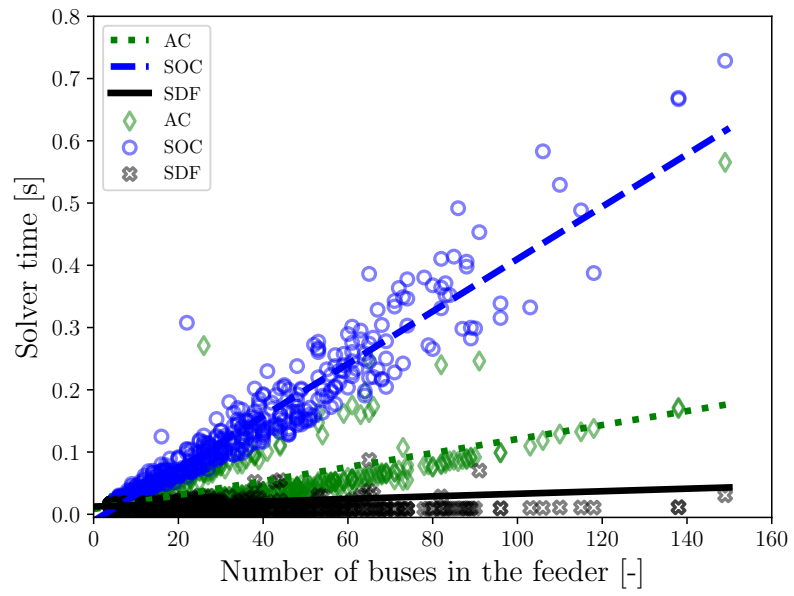

Fig. 6: Solver times for the different simulations, OPF problem

TABLE VI: Parameters for trend lines of AC, SOC and SDF solver times in Fig. 6

\begin{tabular}{ccccc}
\hline $\mathrm{f}(\mathrm{y})$ & $\mathrm{x}$ & $p_{1}$ & $p_{0}$ & $E$ \\
\hline AC & Number of buses & $1.12 \mathrm{E}-3$ & $8.50 \mathrm{E}-3$ & 0.537 \\
SOC & Number of buses & $4.21 \mathrm{E}-3$ & $-9 \mathrm{E}-3$ & 0.473 \\
SDF & Number of buses & $2.05 \mathrm{E}-4$ & 0.013 & 0.032 \\
\hline
\end{tabular}


Finally, the optimality gaps are displayed in Fig. 7. Despite the quite accurate estimation of bus voltages, the errors along the feeders sum up, and it can be observed that the difference in the objective is more substantial and increases with increasing voltage unbalance.

It is again clear that the SDF is more accurate than the SOC, but while the accuracy on bus voltages makes the SDF probably suitable for PF calculations and other applications where voltage magnitude is the crucial factor, it is more questionable whether that is still true for this OPF problem.

The linear trend line of Fig. 7 shows a SOC line which is shifted upwards with respect to the SDF, although the locations of the data points do not seem to justify it. This is because of 11 outliers in the SOC case (2.2\% of the analyzed feeders), which are not displayed in Fig. 7 for ease of visualization. The outlier feeders have $\Delta \mathrm{Obj}_{\mathrm{SOC}}$ values between $26 \%$ and $98 \%$ and have average properties, such as relatively low degree of unbalance (between 0.05 and $1.10 \%$ ), and average length and number of buses. It is therefore unclear what the reason of the computational instability is.

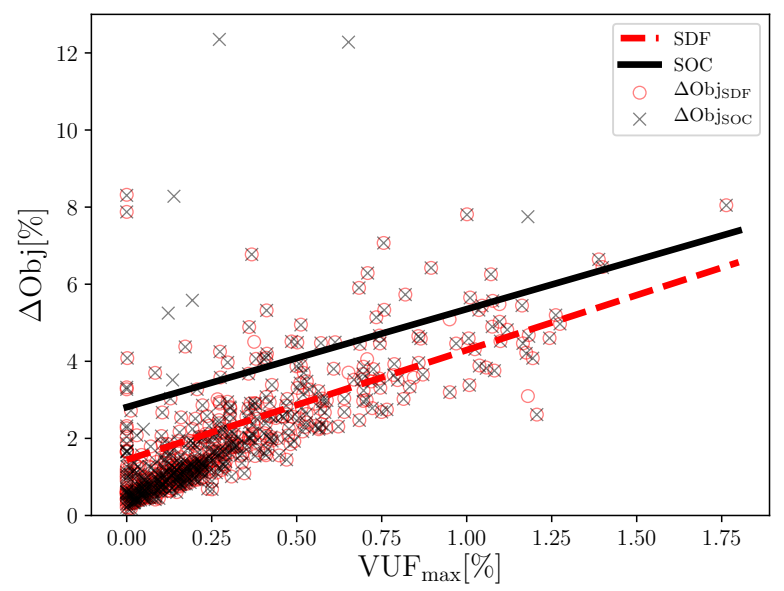

Fig. 7: Optimality gap increase with increasing unbalance, OPF problem

\section{MIXED-INTEGER PROBLEM}

After illustrating that the SDF formulation accurately represents three-phase feeders in continuous variables problems, this section explores its usage in the context of a congestion mitigation strategy for distribution networks in the form of a multiperiod mixed-integer problem (MIP).

The increasing number of EV charging on residential feeders makes the occurrence of undervoltage and overcurrent events more likely. It is assumed that residential users agree to have a "flexible connection" and limit their power consumption below a contractually determined threshold upon request, in exchange for economic advantages (e.g., reduced electricity tariffs). The request takes place on the day before, if risk for congestion is forecasted.

To decide which customers should reduce their power and when, OPF calculations are performed, with the objective of maximizing user comfort, while respecting the voltage and thermal limits of the feeder.

In this work, a "user-constraint-free" power reduction scheme is illustrated, which means that there is no limit to the duration for which the users are asked to provide flexibility. Such conditions cannot be imposed to users in real-life, but it is a good example to assess the suitability of the formulations to solve large MIPs.

The aim of this section is to analyze whether it is possible to solve such a problem with sufficient accuracy and in acceptable time with the simplified formulations presented in the previous sections and whether the solution results in congestion-free scenarios which are AC feasible.

\section{A. Description of the problem}

The OPF problem involves a set of users $\mathcal{U}$, connected on one or three phases of a feeder. The time window analyzed consists of a set of timesteps $\mathcal{T}$.

\section{Problem 4: MIP - BIM and BFM}

Variables:

(8) in the BIM, (8), (13) in the BFM

$$
s_{u, t} \in\{0,1\} \quad \forall u \in \mathcal{U}, t \in \mathcal{T}
$$

Subject to:

$$
\begin{aligned}
& (9)-(12),(21)-(23) \text { in the BIM } \\
& (9),(12),(14)-(17) \text { and }(21)-(23) \text { in the BFM } \\
& S_{u, t, c}=S_{u}^{g t d} \cdot\left(1-s_{u, t}\right)+s_{u, t} \cdot S_{u, t, c}^{f x} \\
& \qquad \forall t \in \mathcal{T}, u \in \mathcal{U}, c \in \phi
\end{aligned}
$$

With objective:

$$
\operatorname{maximize} \sum_{u \in \mathcal{U}} \sum_{t \in \mathcal{T}} s_{u, t}
$$

$s_{u, t}$ is the binary variable that determines whether a user $u$ is required to reduce his demand at timestep $t$. If demand is reduced $\left(s_{u, t}=0\right)$, then the user demand is set to its guaranteed power $S_{u}^{g t d}$ using (25).

$S_{u}^{g t d}$ represents a contractually guaranteed demand, to which user $u$ is entitled even in case of congestion.

If the user is not required to reduce his demand, $s_{u, t}=1$ and he is entitled to consume the forecasted amount $S_{t, c}^{f x}$ to the full extent.

The objective (26) consists of reducing user demand as little as possible, maximizing user comfort.

\section{B. Results}

For this simulation, a set of 100 feeders is chosen which present significant overcurrent and undervoltage problems when a large fleet of charging EVs is added to the current customer demand, such that they would not have a feasible solution for the OPF problem presented in Section III-C.

The solver used for the mixed-integer nonlinear problem (MINLP), based on the AC formulation, is Juniper 0.5.3 [30], 
which relies on Gurobi as MIP solver and Ipopt as nonlinear solver. The feasibility-pump feature of Juniper is activated.

For the mixed-integer linear problem (MILP), based on the SDF formulation, and the mixed-integer second-order cone problem (MISOCP), Gurobi 9.0.1 is chosen, as it proved one order of magnitude faster than Cplex and Mosek in presence of integer variables.

Table VII provides a comparison of the objective function value and computation time for six selected feeders, to help understand the problem. The \#bin columns of the table indicate the number of binary variables, which corresponds to the number of time steps multiplied by the number of participating users $\mathcal{U}$ in the feeder. It also corresponds to the maximum possible value of the objective function, achievable when power reduction is not necessary.

As the solver time for the AC formulation is very long, only 24 time steps are analysed, corresponding to 6 hours with a 15 minutes resolution. A computational time limit is set at 3600 s, after which the solver is stopped. If the time limit is reached, it is indicated in the table as limit. It should be noted that a solution always exists, as in the worst-case scenario the problem allows to curtail all users at all time steps.

If the chosen time instances present no congestions (Feeder 4 and 5), all formulations could retrieve a solution. However, the MINLP and the MISOCP are respectively 4 and 3 orders of magnitude slower than the MILP. In Feeder 1 and 6, where the number of binary variables is limited, a solution is found for the MINLP before the time limit is reached, but with a speed 2-4 orders of magnitude slower than in MILP and MISOCP.

In all feeders, the objective of the MISOCP is greater or equal to that of the MILP. While the MISCOP formulation is able to find solutions closer to the MINLP solution in many instances, such as feeder 5 , this observation cannot be generalized. This is displayed in Feeder 6, where in a very congested scenario both MINLP and MILP formulations perform more reduction actions than the MISOCP.

In the cases where a solution for the MINLP is not found before the time limit, AC feasibility is checked by using the power reduction obtained by the MILP and MISOCP as input of the AC PF problem. To make sure that the congestion mitigation method is as effective as possible on a large-scale real-data set up, slightly tighter thermal and voltage limits (e.g., $99 \%$ of the actual thermal rating, $98 \%$ of the minimum allowed voltage) are considered when performing the MILP and MISOCP calculations, w.r.t. the MINLP case. This can cause extra power reduction with respect to the optimal MINLP value, but it safeguards grid constraints. Under these stricter conditions, all 100 MILP solutions are AC feasible, while 58 of the MISOCP are not.

Fig. 8 shows the solver time of both MISOCP and MILP for all 100 feeders, in simulations with 96 time steps, which correspond to the full 24 hours with 15 min resolution. A MILP solution is found, on average, in $11.75 \mathrm{~s}$, which is 30 times less than the MISOCP average. This, together with the $\mathrm{AC}$ feasibility problems, makes the SOC formulation inapplicable to this type of MIP problem, while the SDF performs reasonably well.

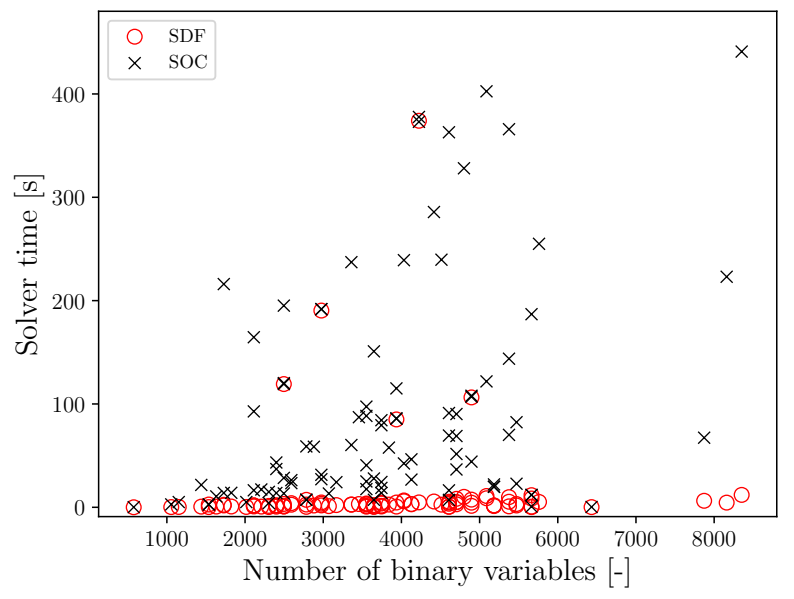

Fig. 8: Solver times for MIP problem, 96 time steps, SDF and SOC formulations

\section{CONClusions}

This paper investigates the accuracy and computational effort of different formulations of unbalanced power flow equations applied to low voltage systems. Simulations are performed on a large number of real-life Belgian feeders, and address different problem types: power flow, OPF with continuous variables and a mixed-integer problem. The analysis takes into account how the number of nodes and degree of voltage unbalance affect the results.

It is shown that the computational time increases with the number of buses in the feeders, especially in the nonlinear

TABLE VII: MIP problem results with all formulations for 6 selected feeders, 24 time steps

\begin{tabular}{l|c|c|c||c|c|c|c|c|c|c}
\hline & \multicolumn{3}{c}{ Feeder 1 } \\
& objective [-] & \#bin [-] & time [s] & objective [-] & \#bin [-] & time [s] & objective [-] & \#bin [-] & time [s] \\
\hline MINLP & 120 & 144 & 127.309 & limit & 1272 & limit & limit & 1296 & limit \\
MISOCP & 120 & 144 & 0.057 & 1272 & 1272 & 35.626 & 1293 & 1296 & 5.561 \\
MILP & 120 & 144 & 0.010 & 1248 & 1272 & 1.896 & 1289 & 1296 & 0.263 \\
\hline \hline & Feeder 4 \\
\hline & objective [-] & \#bin [-] & time [s] & || & objective [-] & \#bin [-] & time [s] & objective [-] & \#bin [-] & time [s] \\
\hline MINLP & 1296 & 1296 & 45.700 & 1080 & 1080 & 34.472 & 126 & 912 & 43.234 \\
MISOCP & 1296 & 1296 & 3.791 & 1080 & 1080 & 6.327 & 761 & 912 & 9.981 \\
MILP & 1296 & 1296 & 0.083 & 1079 & 1080 & 0.138 & 126 & 912 & 0.174 \\
\hline
\end{tabular}


formulations. Furthermore, there is a correlation between unbalance degree $\left(\mathrm{VUF}_{\max }\right)$ and loss of accuracy, for both the SDF and SOC formulations. Nevertheless, the voltage magnitude accuracy $(\Delta \mathrm{U})$ is remarkable for all analyzed feeders, especially in the SDF case. The SDF formulation also presents lower optimality gap values w.r.t. the SOC.

In general, the trade-off between accuracy and computational effort is interesting in the SDF case, which finds a solution approximately 5 times faster than using the full $\mathrm{AC}$ formulation and 10 to 20 times faster than with the SOC. While tightening bounds could result in a speed improvement for the SOC case, it seems less appealing to resort to this strategy than to simply make use of the more accurate SDF.

Finally, a mixed-integer problem is presented, in which using the SDF formulation appears to be the only possible solution due to the feasibility problems of the SOC-based problem and the considerably higher computational time to solve both the MISOCP and MINLP.

\section{ACKNOWLEDGMENTS}

The authors would like to thank Joris Lemmens and Vincent Vancaeyzeele from Fluvius, Frederik Geth from CSIRO and Sander Claeys from KU Leuven for the valuable discussions and contributions.

\section{REFERENCES}

[1] "Electric vehicle conductive charging system - Part 1: General requirements," IEC 61851-1:2017, 2017.

[2] J. G. Robertson, G. P. Harrison, and A. R. Wallace, "OPF techniques for real-time active management of distribution networks," IEEE Trans. Power Syst., vol. 32, no. 5, pp. 3529-3537, 2017.

[3] L. Gan and S. H. Low, "Convex relaxations and linear approximation for optimal power flow in multiphase radial networks," in Power Syst. Comp. Conf (PSCC), Wroclaw, Poland, 2014, pp. 1-9.

[4] C. Zhao, E. Dall'Anese, and S. Low, "Convex relaxation of OPF in multiphase radial networks with delta connections," in 10th IREP Symposium on Bulk Power System Dynamics and Control, Espinho, Portugal, 2017, pp. 1-7.

[5] D. K. Molzahn, B. C. Lesieutre, and C. L. DeMarco, "Approximate representation of ZIP loads in a semidefinite relaxation of the OPF problem," IEEE Trans. Power Syst., vol. 29, no. 4, pp. 1864-1865, 2014.

[6] D. K. Molzahn and I. A. Hiskens, "A survey of relaxations and approximations of the power flow equations," in Foundations Trends Electr. Energy Syst., vol. 4, no. 1-2, 2019, pp. 1-221.

[7] F. Capitanescu, J. Martinez Ramos, P. Panciatici, D. Kirschen, A. Marano Marcolini, L. Platbrood, and L. Wehenkel, "State-of-theart, challenges, and future trends in security constrained optimal power flow," Electr. Power Syst. Res., vol. 81, no. 8, pp. 1731 - 1741, 2011.

[8] J. F. Franco, L. F. Ochoa, and R. Romero, "AC OPF for smart distribution networks: An efficient and robust quadratic approach," IEEE Trans. Smart Grid, vol. 9, no. 5, pp. 4613-4623, 2018.

[9] K. W. Hedman, S. S. Oren, and R. P. O'Neill, "A review of transmission switching and network topology optimization," in IEEE PES General Meeting, Detroit, Michigan, 2011, pp. 1-7.

[10] M. S. Andersen, A. Hansson, and L. Vandenberghe, "Reducedcomplexity semidefinite relaxations of optimal power flow problems," IEEE Trans. Power Syst., vol. 29, no. 4, pp. 1855-1863, 2014.

[11] B. Mahdad, T. Bouktir, and K. Srairi, "A three-phase power flow modelization: A tool for optimal location and control of FACTS devices in unbalanced power systems," in IECON 2006 - 32nd Annual Conference on IEEE Industrial Electronics, Paris, France, 2006, pp. 2238-2243.

[12] C. Coffrin, D. Gordon, and P. Scott, "NESTA, the NICTA energy system test case archive," pp. 1-26, 2014. [Online]. Available: http://arxiv.org/abs/1411.0359
[13] F. Postigo Marcos, C. Mateo, T. Gomez, B. Palmintier, B.-M. Hodge, V. Krishnan, F. de Cuadra, and B. Mather, "A review of power distribution test feeders in the United States and the need for synthetic representative networks," Energies, vol. 10, p. 1896, (11) 2017.

[14] Powermodelsdistribution.jl. [Online]. Available: https://github.com/lanlansi/PowerModelsDistribution.j1

[15] C. Coffrin, R. Bent, K. Sundar, Y. Ng, and M. Lubin, "Powermodels.jl: An open-source framework for exploring power flow formulations," in Power Syst. Comp. Conf. (PSCC), Dublin, Ireland, 2018, pp. 1-8.

[16] C. Coffrin, H. L. Hijazi, and P. Van Hentenryck, "The QC relaxation: A theoretical and computational study on optimal power flow," IEEE Trans. Power Syst., vol. 31, no. 4, pp. 3008-3018, 2016.

[17] R. C. Dugan and T. E. McDermott, "An open source platform for collaborating on smart grid research," in IEEE PES General Meeting, Detroit, Michigan, 2011, pp. 1-7.

[18] D. M. Fobes, S. Claeys, F. Geth, and C. Coffrin, "Powermodelsdistribution.jl: an open-source framework for exploring distribution power flow formulations," in press.

[19] B. A. Robbins, H. Zhu, and A. D. Domínguez-García, "Optimal tap setting of voltage regulation transformers in unbalanced distribution systems," IEEE Trans. Power Syst., vol. 31, no. 1, pp. 256-267, 2016.

[20] J. F. Marley, D. K. Molzahn, and I. A. Hiskens, "Solving multiperiod OPF problems using an AC-QP algorithm initialized with an SOCP relaxation," IEEE Trans. Power Syst., vol. 32, no. 5, pp. 3538-3548, 2017.

[21] D. Gayme and U. Topcu, "Optimal power flow with large-scale storage integration," IEEE Trans. Power Syst., vol. 28, no. 2, pp. 709-717, 2013.

[22] B. Kocuk, S. S. Dey, and X. A. Sun, "New formulation and strong MISOCP relaxations for AC optimal transmission switching problem," IEEE Trans. Power Syst., vol. 32, no. 6, pp. 4161-4170, 2017.

[23] R. A. Jabr, R. Singh, and B. C. Pal, "Minimum loss network reconfiguration using mixed-integer convex programming," IEEE Trans. Power Syst., vol. 27, no. 2, pp. 1106-1115, 2012.

[24] "Environment compatibility levels for low frequency conducted disturbances and signalling in public low-voltage power supply systems," IEC 61000-2-2:2002, EMC Part 2-2, 2002.

[25] "Voltage characteristics of electricity supplied by public electricity networks," EN 50160:2010, 2010.

[26] M. Vanin, F. Geth, R. D'hulst, and D. Van Hertem, "Electric vehicles charging in unbalanced belgian low-voltage grids: a case study," in CIRED Workshop, Ljubljana, Slovenia, 2018, pp. 1-4.

[27] C. Mateo, G. Prettico, T. Gómez, R. Cossent, F. Gangale, P. Frías, and G. Fulli, "European representative electricity distribution networks," Int. J. Electr. Power Energy Syst., vol. 99, pp. 273 - 280, 2018.

[28] A. Wächter and L. Biegler, "On the implementation of an interiorpoint filter line-search algorithm for large-scale nonlinear programming," Math. Program., vol. 106, no. 1, pp. 25-57, 2006.

[29] HSL. a collection of fortran codes for large scale scientific computation. [Online]. Available: http://www.hsl.rl.ac.uk/

[30] O. Kröger, C. Coffrin, H. Hijazi, and H. Nagarajan, "Juniper: An opensource nonlinear branch-and-bound solver in julia," in Integration of Constraint Programming, Artificial Intelligence, and Operations Research, 2018, pp. 377-386. 